\title{
When do learners shift from habitual to agenda-based processes when selecting items for study?
}

\author{
Robert Ariel • John Dunlosky
}

Published online: 8 November 2012

(C) Psychonomic Society, Inc. 2012

\begin{abstract}
Learners presumably attempt to allocate their study time to maximize reward, yet in some contexts, their study choices are driven by reading biases that would not maximize reward. For instance, when presented with items in a horizontal array that are worth different values if correctly recalled, learners will often first select the leftmost item (i.e., a reading bias), even when it is associated with the lowest value. In four experiments, we investigated the degrees to which various factors cause learners to shift to agenda-based regulation. On each trial, participants were presented with three cues and a point value $(1,3$, or 5$)$ for each. The participants could select any cue for study (in which case, its target would be presented) in any order. In Experiment 1, participants either selected items for study under time pressure or were given unlimited time to select items. Not limiting selection time increased the likelihood that higher-valued items would be prioritized for study, but reading biases still influenced item selection. In Experiment 2, participants could select only one item per trial, and higher-valued items were prioritized even more for study, but not exclusively so. In Experiments 3 and 4, we ruled out a lack of motivation and inaccurate task beliefs as explanations for why participants would sometimes choose lower-valued items. The results demonstrate the influence of a pervasive reading bias on learners' item selections, but as importantly, they show that a shift toward agenda use occurs when habitual responding cannot maximize reward.
\end{abstract}

Keywords Self-regulated study · Item selection ·

Agenda-based regulation $\cdot$ Habitual responding

R. Ariel $(\bowtie)$

School of Psychology, Georgia Institute of Technology,

Atlanta, Georgia 30313

e-mail: robert.ariel@psych.gatech.edu

J. Dunlosky

Psychology Department, Kent State University,

Kent, OH, USA
When studying for an upcoming exam, learners may make a variety of decisions about how to allocate their study time across the to-be-learned materials. These include deciding (a) the order in which to select items for study, (b) how long to study an item before moving on to the next one, and (c) when to terminate a study session (Dunlosky \& Ariel, 2011b). Decades of research on study time allocation have highlighted a variety of factors that influence these decisions, including the reward associated with learning items (Ariel, Dunlosky, \& Bailey, 2009; Dunlosky \& Thiede, 1998; Soderstrom \& McCabe, 2011), the subjective difficulty of learning items (for reviews, see Dunlosky \& Ariel, 2011 b; Son \& Metcalfe, 2000), a learner's intrinsic motivation (Pintrich, 2000; Pintrich \& De Groot, 1990), and even reading habits (Ariel, Al-Harthy, Was, \& Dunlosky, 2011; Dunlosky \& Ariel, 2011a).

According to the agenda-based regulation (ABR) framework of study-time allocation, learners' study decisions are influenced by two qualitatively different kinds of processes: agenda-based and habitual (Dunlosky \& Ariel, 2011b). Agenda-based processes involve learners constructing an agenda - or simple plan - that they use to regulate their study (Ariel et al., 2009; Winne \& Hadwin, 1998). Learners construct agendas in response to environmental conditions to achieve their learning goals efficiently (Thiede \& Dunlosky, 1999). Consider a learner who wants to excel on an upcoming exam. This learner can maximize the likelihood of performing well by constructing an agenda that prioritizes items for study that are likely to return the highest reward (Castel, 2007). This agenda-driven learner will then compare potential study items to the criteria of this agenda and select items for study that meet these criteria.

In contrast to agenda-based processes, habitual processes involve the task environment activating a prepotent or overlearned response (Dunlosky \& Ariel, 2011b). To illustrate the influence of a habitual process on learners' study decisions, consider an experiment from Ariel, Al-Harthy, Was, 
and Dunlosky (2011). Native English readers and native Arabic readers completed a task presented in their native language in which they were allowed to select easy, moderately difficult, and difficult cue-target items for study. On each trial, a cue word from each difficulty level was presented and ordered from left to right in an array; the order of difficulty was counterbalanced across trials. The difficulty of each item (i.e., easy, moderate, or difficult) was presented above each cue. Although the best strategy would be to choose the easiest item first on each trial (Metcalfe, 2009; Metcalfe \& Kornell, 2005; Price \& Murray, 2012), the majority of participants failed to construct this agenda. Instead, Native English readers preferred to first select items in the left position of each array, and native Arabic readers preferred to first select items in the right position.

The notion that self-regulated study can be influenced by either agenda-based or habitual processes is consistent with theories of self-regulation in other psychological domains (Baumeister \& Heatherton, 1996; Metcalfe \& Mischel, 1999) and with theories of a wide range of phenomena, including emotions (Teasdale, 1999; van Reekum \& Scherer, 1997), persuasion (Chaiken, 1980; Petty \& Cacioppo, 1986), stereotyping (Devine 1989), and reasoning (De Neys, 2006; Evans, 2003). These theories claim that cognitive processing can operate in either a voluntarily controlled or an automatic mode (for a review, see Evans \& Frankish, 2009). In the present experiments, we evaluated the interplay between these two modes of regulation and how they affect the order in which items are selected for study. More specifically, we evaluated the question, when do learners shift away from habitual responding and construct an agenda to regulate their study?

Given that people tend not to construct agendas during problem solving unless they are pressured to do so by the environment (Hayes-Roth \& Hayes-Roth, 1979; Waldron, Patrick, \& Duggan, 2011), we suspect that in most contexts learners do not spontaneously construct agendas, and instead that study decisions are largely biased by reading habits. Learners may avoid constructing agendas and default to selecting items on the basis of reading habits for a variety of reasons. First, selecting items on the basis of reading habits would be less cognitively demanding than developing an agenda and maintaining it in working memory while executing it. Second, agenda-based regulation can be timeconsuming, because learners have to devote time to developing the agenda and searching the task environment for items that meet the criteria of selection outlined by that agenda. In contrast, selecting items on the basis of reading order can be implemented quickly because it involves minimal search. Finally, selecting items on the basis of reading habits can be relatively effective in many contexts, such as when learners have unlimited time to learn material and their goal is to learn everything.
To summarize, learners' decisions about the order in which to study items are often influenced by reading habits (Ariel et al., 2011), which raises the following question: When do learners construct agendas and shift away from this kind of habitual responding? To answer this question, we evaluated two hypotheses that are relevant to whether and when learners will construct agendas, and thus rely less on habitual responding. Both hypotheses focus on the critical role of task constraints, but the first concerns whether the task constraints are likely to trigger agenda construction, and the second concerns whether learners can construct an agenda given the particular task constraints. According to the first hypothesis, environmental factors must be present that decrease the effectiveness of achieving learning goals through habitual responding. When the task environment is altered to limit the effectiveness of using habitual processes to make study decisions, learners should be more likely to reflect on alternative strategies to meet their learning goals efficiently. According to this hypothesis, when the expected gains to performance as a result of investing the additional time to construct and execute an agenda outweigh the efficiency gained from just selecting items on the basis of reading order, learners should be more likely to use agenda-based processes to make their study decisions (Waldron et al., 2011).

According to the second hypothesis, the learner must have sufficient time to construct an agenda. Research examining the effects of time pressure on reasoning and decision making indicates that people typically rely on quick, heuristic-based reasoning instead of more analytical processes when the time to make decisions is limited (Evans \& Curtis-Holmes, 2005; Roberts \& Newton, 2002; Schroyens, Schaeken, \& Handley, 2003). This hypothesis predicts that time pressure during item selection will discourage agenda-based regulation and instead encourage learners to use quicker, less resource-demanding (i.e., habitual) processes.

To evaluate these hypotheses, we investigated the degrees to which agenda-based regulation is triggered by various factors in the task environment, such as the time available to select and study items (Exp. 1), the number of items that participants are allowed to study on each trial (Exps. 2-4), and monetary incentives for performance (Exp. 3). On each trial, three items were presented, ordered from left to right in a stimulus array, and all of the items were similar in their normative difficulty to learn. The items were slated as having a low reward value (1 point), a moderate reward value ( 3 points), or a high reward value ( 5 points). The three cues were presented in the interface presented in Fig. 1. To study a response, participants clicked on the button (labeled with "?") in the box below its corresponding cue, and the response would be presented in the box. If another button was selected, the current response would disappear and the next response would appear. 
Fig. 1 Illustration of a typical study trial for the 1-3-5 order condition. Participants could select Spanish translations for study by clicking on the question mark button positioned below the corresponding English word that they desired to study. When a translation was selected for study, it was presented

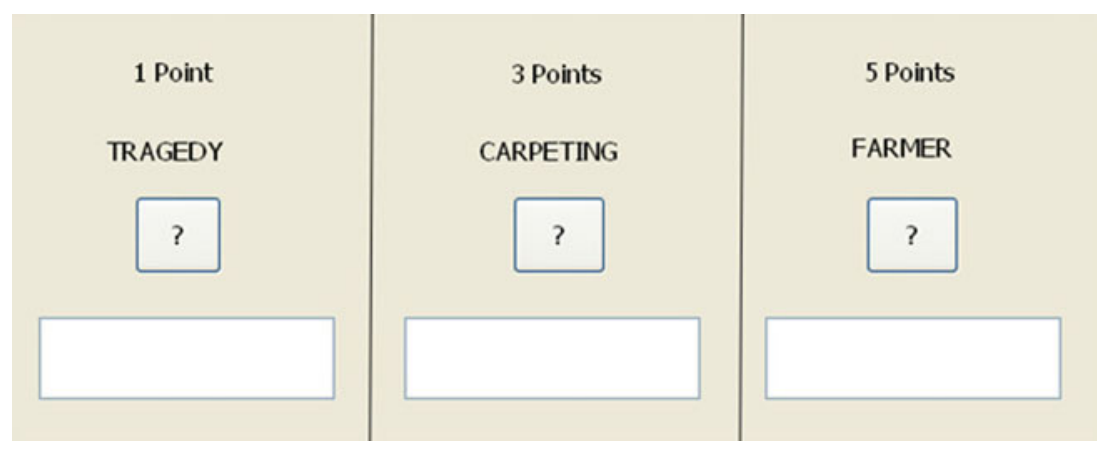

We chose to manipulate the reward associated with learning items because people typically allocate more effort to learning items with a high (vs. lower) reward (Castel, 2007; Castel, Balota, \& McCabe, 2009; Castel, Benjamin, Craik, \& Watkins, 2002; Dunlosky \& Thiede, 1998; Kahneman \& Peavler, 1969; Soderstrom \& McCabe, 2011), and reward can even override the influence of item difficulty on study decisions (Ariel et al., 2009). Thus, when studying items that vary in reward for learning, an obvious strategy to maximize performance would be to construct an agenda to prioritize the high-reward items for study first.

\section{Experiment 1}

In Experiment 1, we manipulated the time available to select and study items on each trial. Across multiple trials, the participants selected items for study from a three-item array in which items were ordered from left to right as a function of item reward. The orderings of items as a function of reward were counterbalanced across trials. In the restricted-total-time group, participants had $5 \mathrm{~s}$ on each trial to select and study items, and hence, they had limited time to construct and execute an agenda. This restricted-total-time group was compared to the restricted-study-time group, who were given unlimited time to make their first study decision, but the amount of time that they had to study items on each trial was restricted to $5 \mathrm{~s}$. This time began only after participants had made their first selection. Our main question for this experiment was, would limiting the time to study items pressure learners to construct an agenda to select highreward items first when they were not pressured to decide quickly? We will now consider three answers to this question and the predicted outcomes relevant to each one.

First, the effects of reading habits on study decisions may be relatively potent, and thus powerful enough to bias study decisions regardless of other factors in the task environment, such as item reward and the time available to study items. Reading habits have a powerful effect on a variety of cognitive and perceptual motor processes (for relevant references, see Ariel et al., 2011). Given that study materials are text-based in nature, reading habits may have a particularly strong effect on study decisions that is unlikely to diminish in response to other factors in the study task environment. If such a reading bias were influential in the present experiments, one would expect participants to select items in the left position of the array first for study on each trial, regardless of the presentation order of the items or of other aspects of the task environment.

A second possibility is that learners will always select high-reward items first for study, regardless of other factors in the task environment. When people encounter items that vary in rewards for learning, they typically invest more resources in learning the items that are high in reward (as we discussed above). Thus, processing variations in reward alone may be enough to trigger strategic behavior. If so, participants should always select the high-reward (5-point) item first for study, regardless of its position in the array and the time available to study the items.

A final possibility is based on the two hypotheses described above that predict when learners will shift from habitual to agenda-based regulation. Namely, there must be pressure to behave strategically from the task environment (e.g., participants cannot learn all of the items on any given trial, and basing choices on reading order will not yield the largest reward), and learners must have sufficient time to construct an agenda. In the absence of either condition, learners will default to selecting items on the basis of reading order. If so, an interaction would be expected between time allowed and item reward, with learners selecting the leftmost item first (i.e., reading order) when there is pressure to select items quickly (restricted-totaltime group), but more often selecting the high-reward item first (i.e., agenda-based decision) when time for making the first selection is unlimited (restricted-study-time group).

\section{Method}

Participants A group of 102 students from Kent State University (KSU) participated for credit in an Introductory Psychology course. A 6 (presentation order: 1-3-5, 1-5-3, $3-1-5,3-5-1,5-1-3$, or $5-3-1) \times 3$ (item reward: 1,3 , or 5 points) $\times 2$ (time allowed: restricted total time vs. restricted study time) mixed factorial design was used, with Presentation Order and Item Reward as within-subjects factors. Participants 
were randomly assigned to either the restricted-total-time group $(n=51)$ or the restricted-study-time group $(n=51)$.

Materials and procedure A set of 48 moderately difficult English-Spanish vocabulary pairs (e.g., prophecy-vatcinio) from Metcalfe (2002) were adapted for use in this experiment. An additional six English-Spanish pairs were added to this list, to create a total of 54 moderately difficult pairs. All pairs were randomly assigned a reward value of 1,3 , or 5 points at the beginning of the experiment.

The participants were instructed that they would be learning English-Spanish vocabulary pairs and that they would receive points for correctly recalling Spanish translations on the final test. The participants were told that some translations were worth 1 point, some were worth 3 points, and others were worth 5 points. Participants in the restricted total-time-group were instructed that they would have $5 \mathrm{~s}$ to select and study items on each trial, and participants in the restricted-studytime group were instructed that they had unlimited time to make their first choice for study on each trial. However, after selecting the first item, they had $5 \mathrm{~s}$ to study that item and any subsequent items selected. All of the participants were instructed that they were free to allocate their study time however they wished and that they would be tested on all of the Spanish translations after they had finished studying.

An illustration of a typical study trial is displayed in Fig. 1. On each study trial, three English words were presented on the screen, positioned in order from left to right. The order of presentation of each item was manipulated within participants so that each participant experienced each possible order of reward $(1-3-5,1-5-3,3-1-5,3-5-1,5-$ $1-3$, and 5-3-1) three times across trials, for a total of 18 trials. The 18 trials consisted of three blocks of six trials (Trials 1-6, 7-12, and 13-18). The presentation order during these blocks was constrained so that participants experienced each possible order of reward within each block. However, the order that these conditions occurred in within each block was randomized. All items were randomly assigned to the presentation order conditions and randomly assigned to each block for each participant. On each study trial, above each English word, the point value (1, 3, or 5 points) of learning a particular word's Spanish translations was presented. Participants could view the Spanish translation of a given word by clicking on a button positioned below each English word. When the participants selected this button, the Spanish translation was presented in text field below the English equivalent. The translation remained on the screen until participants selected a different translation or until time expired for that trial. If participants selected a different translation, that word was presented below its English equivalent and the current translation was removed. After 18 trials, the participants were tested: Each English word was presented, and they were prompted to type its translation.

\section{Results and discussion}

To evaluate the a priori predictions, we examined the order of item selection across trials as a function of array position (left, middle, or right). We will present only participants' first choice preferences across trials, because these data were most relevant to examining whether learners constructed an agenda to prioritize high-reward items for study or whether their study decisions were biased by reading habits. After examining item selection, we will briefly report the self-paced study data. Recall performance was not relevant to evaluating the central hypotheses, but these data are available from the first author. All effects reported as significant had $p<.05$.

Item selection The proportions of trials on which participants selected items in the left, middle, and right positions of the array first for study on each trial were computed as a function of presentation order. The means are presented in Fig. 2. Presentation order condition is presented on the $x$ axis, and bars reflect the position selected from the array (left, middle, or right). The data for the restricted-total-time group are presented in the left panel, and the data for the restricted-study-time group are presented in the right panel. Comparisons of selections for items in the left position and in the high-reward position of the array (i.e., where the 5point item was located) are central for evaluating the degree to which study decisions are influenced by reading habits versus an agenda to study the 5-point items. Because both habitual and agenda-based processing predict that participants would select items in the left position of the array in the 5-1-3 and 5-3-1 conditions, these conditions were excluded from all analyses but are included in each figure. By contrast, the key conditions for examining the influence of habitual versus agenda-based processes are those in which the highest-valued item was not in the left position, and hence these conditions are the critical ones for evaluating the central hypotheses. These conditions are highlighted in Fig. 2 with an asterisk and bracket. Scanning Fig. 2 from left to right, it is evident that the restricted-total-time group selected a higher proportion of items in the left position of the array first (black bars), which is consistent with a habitual reading bias. The restricted-study-time group also selected a high proportion of items in the left position of the array (black bars); however, they preferred selecting 5-point items more than did the restricted-total-time group when these items were presented in middle (gray bars in the 35-1 and 1-5-3 conditions) and right (white bars in the 3-15 and $1-3-5$ conditions) positions of the array. 


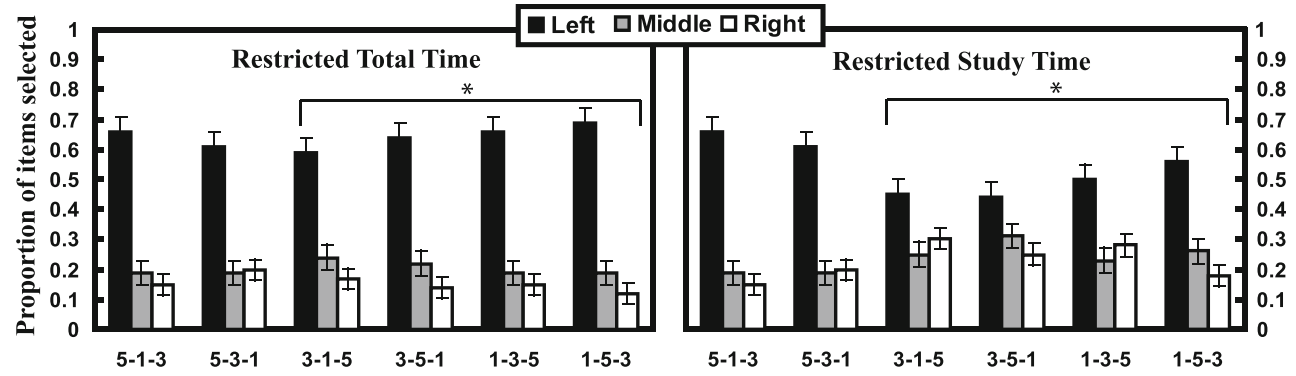

Fig. 2 Proportions of items chosen first for study in the left, middle, and right positions of the array when the time available to select and study items was restricted (left panel) and when time was restricted only during study (right panel) in Experiment 1. Presentation order conditions are presented on the $x$-axis: $1=1$ point, $3=$ points, $5=5$ points. Only the order conditions in brackets are relevant for contrasting the influences of agenda-based and habitual processes. Error bars represent standard errors of the means
These observations were confirmed by a 2 (position selected: left vs. high reward) $\times 2$ (time allowed: restricted total time vs. restricted study time) $\times 4$ (presentation order: $3-1-5,3-5-1$, $1-3-5$, or 1-5-3) analysis of variance (ANOVA). The ANOVA revealed effects of presentation order, $F(3,98)=2.97, M S E=$ $.07, \eta_{\mathrm{p}}{ }^{2}=.08$, and position selected, $F(1,100)=47.52$, MSE $=$ $22.67, \eta_{\mathrm{p}}{ }^{2}=.32$. Most importantly, the effect of position selected was qualified by a Position Selected $\times$ Time Allowed interaction, $F(1,100)=7.99, M S E=3.57, \eta_{\mathrm{p}}{ }^{2}=07$. This interaction occurred because participants in the restrictedtotal-time group selected a higher proportion of items in the left position of the array first for study (collapsed across presentation orders: $M=.65, S E=.04$ ) than did the restrictedstudy-time group (collapsed across presentation orders: $M=$ $.49, S E=.04), t(100)=2.67$. Thus, reading habits biased participants' choices more when time was restricted for constructing an agenda. When time pressure was removed from agenda construction, participants behaved more strategically and selected more high-reward items for study (restricted study time, $M=.29, S E=.03$; restricted total time, $M=.18, S E=.02), t(100)=2.60$.

Despite this increased preference for selecting high-reward items, participants' study decisions in the restricted-study-time group were influenced more by reading habits than by item reward, in that they preferred selecting items in the left position of the array more than high-reward items in other positions, $t \mathrm{~s}>1.53$. One reason that time pressure (with unlimited time for agenda construction) may not have completely overridden the influence of reading habits is that some participants might have believed that $5 \mathrm{~s}$ was enough time to study all three items. Although this is possible, the patterns of item selection were inconsistent with this belief, because on average, participants did not select all three items for study on each trial (restricted total time group, $M=2.21, S E=0.14$; restricted study time group, $M=1.97, S E=0.13$ ).

Self-paced study It is possible that learners neglected to consider the order in which they selected items for study, yet still allocated more time to items selected for study differentially based on item reward. To evaluate this possibility, we computed the mean self-paced study times, in seconds, for the first three items selected for study on a given trial as a function of item reward. The means across individual mean values are presented in Table 1. In Experiment 1 and all subsequent experiments, we found no effects of presentation order on self-paced study. Therefore, to simplify the analyses, we collapsed across presentation order conditions in Table 1 and the subsequent ANOVAs. ${ }^{1}$ Most importantly, participants did not allocate time differently to 1-, 3-, and 5-point items. A 2 (item reward) $\times 2$ (time allowed: restricted total time vs. restricted study time) ANOVA revealed only an effect of time allowed, $F(1,100)=42.50, M S E=161.65, \eta_{\mathrm{p}}{ }^{2}=.30$, which occurred because participants in the restricted-study-time group allocated more time to studying items of each reward type than did the restricted-total-time group. This effect was likely due to the restricted-study-time group having additional time available to study items, because the time available to study was independent of the time available for item selection in this group.

\section{Experiment 2}

The results from Experiment 1 confirmed the central hypotheses, because learners selected the higher-valued items more often when ample time was available to construct an agenda and when study time was limited, and hence all items could not be learned. Nevertheless, and rather surprisingly, time pressure during study did not completely override the

\footnotetext{
${ }^{1}$ The mean self-paced study times for 1-, 3-, and 5-point items do not sum to $5 \mathrm{~s}$ because participants on average only selected approximately two items for study on each trial. Mean self-paced study times were computed using only the items selected for study because, if an item was not selected for study, its study time was unavailable. Most importantly, the study times did not differ as a function of point values within the various groups.
} 
Table 1 Mean self-paced study times, in seconds, as a function of point value

\begin{tabular}{|c|c|c|c|}
\hline \multirow[t]{2}{*}{ Group } & \multicolumn{3}{|l|}{ Item Reward } \\
\hline & 1 point & 3 points & 5 points \\
\hline \multicolumn{4}{|l|}{ Experiment 1} \\
\hline Restricted total time & $1.84(0.11)$ & $1.74(0.11)$ & $1.83(0.09)$ \\
\hline Restricted study time & $3.29(0.21)$ & $3.19(0.21)$ & $3.31(0.20)$ \\
\hline \multicolumn{4}{|l|}{ Experiment 2} \\
\hline No choice limit & $4.51(0.77)$ & $4.20(0.73)$ & $3.94(0.65)$ \\
\hline Choice limit & $3.80(0.37)$ & $3.96(0.37)$ & $4.08(0.35)$ \\
\hline \multicolumn{4}{|l|}{ Experiment 3} \\
\hline \multicolumn{4}{|c|}{ No Performance Incentive } \\
\hline No choice limit & $1.56(0.24)$ & $1.55(0.19)$ & $1.68(0.24)$ \\
\hline Choice limit & $1.46(0.22)$ & $1.29(0.17)$ & $2.48(0.53)$ \\
\hline \multicolumn{4}{|l|}{ Performance Incentive } \\
\hline No choice limit & $1.55(0.23)$ & $2.23(0.52)$ & $2.79(0.90)$ \\
\hline Choice limit & $1.66(0.31)$ & $3.39(0.86)$ & $4.50(1.16)$ \\
\hline \multicolumn{4}{|l|}{ Experiment 4} \\
\hline \multicolumn{4}{|l|}{ Low Reward Contrast } \\
\hline No debias & $0.95(0.20)$ & $1.33(0.24)$ & $6.21(1.86)$ \\
\hline Debias & $1.31(0.30)$ & $1.98(0.37)$ & $4.20(0.82)$ \\
\hline \multicolumn{4}{|l|}{ High Reward Contrast } \\
\hline No debias & $1.07(0.21)$ & $1.26(0.24)$ & $5.21(1.27)$ \\
\hline Debias & $1.09(0.30)$ & $1.00(0.24)$ & $7.77(1.61)$ \\
\hline
\end{tabular}

Values are means across individual participants' mean values. Standard errors of the means are in parentheses. In Experiment 4, all groups were given a choice limit, and the values for the high-reward-contrast groups under the 5-point column reflect values for 50-point items

influence of a reading bias. In Experiment 2, we manipulated a different factor of the task environment that we expected would have a stronger influence. Specifically, we manipulated the number of items that participants could select for study on each trial. They could either select as many items as they wanted (no-choice-limit group) or only one item per trial (choice-limit group). The purpose of this manipulation was to increase the cost associated with not constructing an agenda to study high-reward items first. In this context, if participants' study decisions were biased by reading habits, they would often choose to study a lower-valued ( 1 or 3 points) item, even though selecting any item other than the 5-point item was an obviously ineffective strategy.

According to the first central hypothesis, the extra cost associated with choosing low-valued items when there was a choice limit should further increase the likelihood that participants would shift toward using an effective agenda and more often select the 5-point items. Note, also, that given that our intent was to investigate the factors that might trigger agenda-based regulation, in the remainder of the experiments, participants were given ample time to construct an agenda. Thus, any suboptimal regulation could not be attributed to undermining participants' ability to potentially construct an agenda (as per the second central hypothesis, which was confirmed in Exp. 1).

\section{Method}

Participants A group of 43 students from KSU participated for credit in an Introductory Psychology course. A 6 (presentation order) $\times 3$ (item reward) $\times 2$ (choice limit: no choice limit vs. choice limit) mixed factorial design was used, with Presentation Order and Item Reward as within-subjects factors. The participants were randomly assigned to the no-choice-limit $(n=22)$ or choice-limit $(n=21)$ group.

Materials and procedures The same materials and procedures were used as in Experiment 1, with the following two exceptions. First, all participants had unlimited time to select and study items, so that they had sufficient time to construct an agenda. When the participants were finished studying on a trial, they pressed a button in the bottom right corner of the screen for the next trial. Second, they could either select as many items as they wanted on each trial (no-choice-limit group) or select only one per trial (choice-limit group). If a participant in the choice-limit group attempted to select more than one item, a warning message reminded them that they could only select one item per trial.

\section{Results and discussion}

Item selection The proportions of times that participants selected items first for study in each position of the array (left, middle, or right) across presentation orders are presented in Fig. 3. A 2 (position selected: left vs. high-reward position $) \times 2$ (choice limit: no choice limit vs. choice limit) $\times 4$ (presentation order) ANOVA revealed only a Position Selected $\times$ Choice Limit interaction, $F(1,41)=18.02, M S E$ $=8.53, \eta_{\mathrm{p}}{ }^{2}=.31$. This interaction occurred because participants in the no-choice-limit group preferred selecting items in the left position of the array (collapsed across presentation order conditions: $M=.63, S E=.07)$ more than items in the high-reward position (collapsed across presentation order conditions: $M=.20, S E=.05$ ). By contrast, participants in the choice-limit group preferred selecting items in the high-reward position $(M=.47, S E=.06)$ more than items in the left position $(M=.28, S E=.04), t \mathrm{~s}>2.08$. These outcomes are consistent with the hypothesis that limiting the number of choices would trigger agenda construction and reduce the influence of reading habits. 


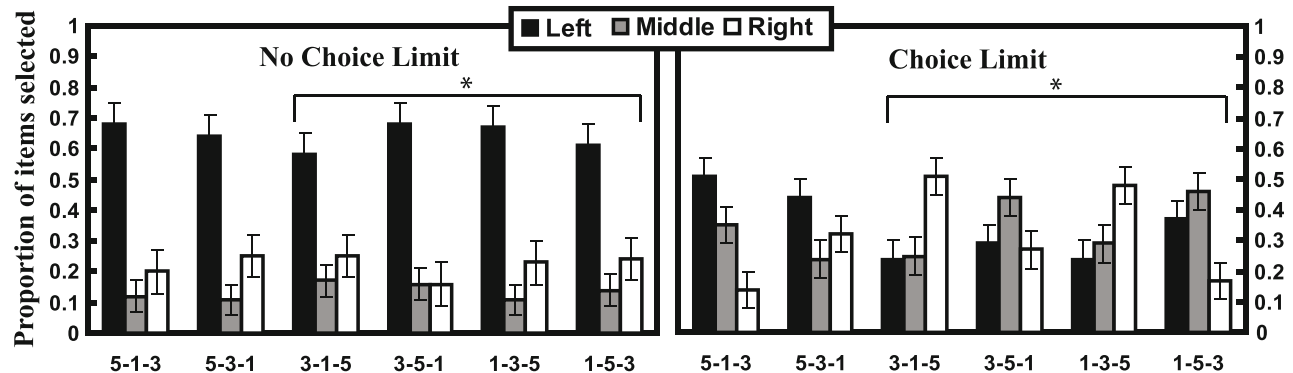

Fig. 3 Proportions of items chosen first for study in the left, middle, and right positions of the array when participants' number of choices was not limited (left panel) and when they were limited to one choice per trial (right panel) in Experiment 2. Presentation order conditions

Self-paced study Mean self-paced study times are presented in Table 1. No effects were significant. Thus, regardless of whether participants selected a high-reward or low-reward item for study, they invested approximately equal amounts of time in studying that item.

\section{Experiment 3}

Consistent with the first hypothesis, limiting the number of items that participants could select for study on each trial pressured learners to prioritize high-reward items first for study. Despite the effectiveness of this manipulation in overriding the influence of reading habits on study decisions, it is somewhat surprising that learners still selected a high proportion of 1- and 3-point items when they could only choose one item for study. In fact, the preference for selecting the highreward items was only around $50 \%$ in the choice-limit group. One might wonder why participants ever select 1- or 3-point items when they will be rewarded less for learning them. In Experiment 3, we evaluated whether this outcome was due to participants lacking the motivation to earn points.

One potential criticism of Experiments 1 and 2 is that the reward that participants received for recalling items on the final test (i.e., points) was arbitrary. It is likely that these points were not intrinsically valuable, and hence, all participants may not have been motivated to maximize reward. Participants who are not motivated to maximize reward may be more susceptible to the influence of habitual processes on their study decisions (e.g., Isbell \& Wyer, 1999; Kool, McGuire, Rosen, \& Botvinick, 2010). Thus, in Experiment 3 , we included an incentive manipulation that served as an extrinsic motivator to participants. Those who received this incentive were instructed that they would receive $\$ 10$ if they earned at least 40 points on the final test. They were also told that their name would be entered in a raffle to receive $\$ 50$, if they earned at least 60 points. Importantly, note that participants could not achieve either goal in the choice-limit are presented on the $x$-axis: $1=1$ point, $3=$ points, $5=5$ points. Only the order conditions in brackets are relevant for contrasting the influences of agenda-based and habitual processes. Error bars represent standard errors of the means

groups if they only selected 1-point items, and the easiest way to achieve the goal was to select 5-point items.

\section{Method}

Participants A group of 92 students from KSU participated for credit in an Introductory Psychology course. A 6 (presentation order) $\times 3$ (item reward) $\times 2$ (choice limit $) \times 2$ (incentive: monetary incentive vs. no incentive) mixed factorial design was used with Presentation Order and Item Reward as within-subjects factors. The participants were randomly assigned to either the choice-limit-with-incentive group ( $n=23)$, the choice-limit-with-no-incentive group ( $n$ $=23)$, the no-choice-limit-with-incentive group $(n=23)$, or the no-choice-limit-with-no-incentive group $(n=23)$.

Materials and procedures The materials and procedure were identical to those in Experiment 3, with the exception that some participants were given a monetary incentive to perform well on the final recall test. Participants in the incentive groups were instructed that for participating in the experiment, their name would be entered in a raffle to win $\$ 50$, and they could receive $\$ 10$ if they earned 40 points on the final test. In addition, they were instructed that if they earned 60 points on the final test, their name would be entered two additional times in a raffle to win $\$ 50$. All of the other instructions were identical to those for the noincentive groups. Participants were paid as described above, and one participant won the raffle as described above.

\section{Results and discussion}

Item selection As is evident from the proportions of items first selected for study (in Fig. 4), incentive appeared to minimally influence item selection, and the effects of choice limit replicated the outcomes from Experiment 2. A 2 

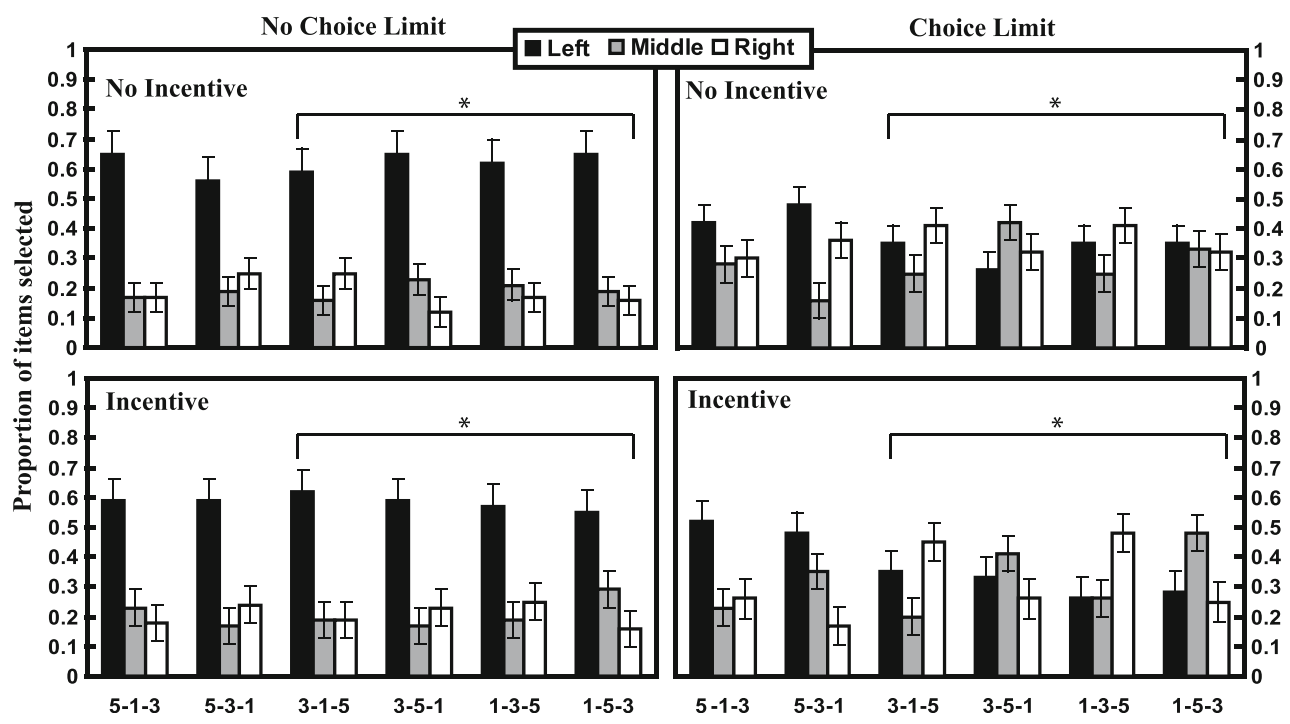

Fig. 4 Proportions of items chosen first for study in the left, middle, and right positions of the array when participants' number of choices was not limited and they were not given a monetary incentive (top left panel), when they were limited to one choice per trial and no incentive was given (top right panel), when they were not limited and they were given a monetary incentive (bottom left panel), and when they were

limited to one choice and an incentive was given (bottom right panel) in Experiment 3. Presentation order conditions are presented on the $x$ axis: $1=1$ point, $3=$ points, $5=5$ points. Only the order conditions in brackets are relevant for contrasting the influences of agenda-based and habitual processes. Error bars represent standard errors of the means

(position selected) $\times 2$ (choice limit) $\times 2$ (incentive $) \times 4$ (presentation order) ANOVA yielded effects for position selected, $F(1,88)=9.64, M S E=3.70, \eta_{\mathrm{p}}^{2}=.10$, and choice-limit group, $F(1,88)=7.73, M S E=0.34, \eta_{\mathrm{p}}{ }^{2}=.08$. Most importantly, these effects were qualified by a Position Selected $\times$ Choice Limit interaction, $F(1,88)=29.66, M S E=$ $11.38, \eta_{\mathrm{p}}{ }^{2}=.25$. Post-hoc analyses indicated that this interaction occurred because (a) for the no-choice-limit groups, preference for selecting items in the left position of the array (collapsed across presentation order conditions: $M=.61, S E$ $=.04$ ) was higher than that for selecting the high-reward position (collapsed across presentation orders: $M=.22, S E=$ $.03)$; by contrast, for the choice-limit groups, preference for selecting items in high-reward position $(M=.42, S E=.03)$ was higher than for selecting items in the left position $(M=$ $.32, S E=.02)$, all $t \mathrm{~s}>1.96$. These findings are consistent with the results from Experiment 2. Interestingly, incentive did not increase preference for selecting high-reward items for study, $F(1,88)=0.65, M S E=0.25$.

Self-paced study The mean self-paced study times are presented in Table 1. A 3 (item reward) $\times 2$ (choice limit) $\times 2$ (incentive) ANOVA revealed significant effects of item reward, $F(2,87)=4.99, M S E=39.43, \eta_{\mathrm{p}}{ }^{2}=.10$, and incentive group, $F(1,88)=5.94, M S E=71.30, \eta_{\mathrm{p}}{ }^{2}=.06$. These main effects were qualified by an Item Reward $x$ Incentive interaction, $F(2,87)=3.32, M S E=17.87, \eta_{\mathrm{p}}{ }^{2}=$ .07 . This interaction occurred because more time was allocated to 3- and 5-point items by the incentive group than by the no-incentive group, $t \mathrm{~s}>1.96$.

In summary, despite the lack of an effect of incentive on item selection, incentive did influence self-paced study times, in that participants allocated more time to studying high-reward items when they were given a monetary incentive to perform well than when they were not given an incentive. One explanation for these differences is that item selection and self-paced study may in part be influenced by different processes. In the present experiments, participants would need to use an agenda to consistently select the 5point items first for study, but self-paced study would not require them to plan to select the 5-point item first on every trial. Instead, on some trials, participants might not use an agenda but, by habit, choose the leftmost item for study. When doing so, they might still perceive the value of the item selected, which itself could influence self-paced study. For instance, Dunlosky and Thiede (1998) proposed that learners stop studying an item when they no longer perceive change in learning for a given period of time (for a similar proposal, see Metcalfe \& Kornell, 2005) and that learners set a threshold for how long they will continue studying an item despite not perceiving any change in learning. Factors like reward (as in the present case) and mastery instructions (Nelson \& Leonesio, 1988) are expected to influence selfpaced study times via changing the placement of this threshold (for details, see Dunlosky \& Thiede, 1998). The idea here is that when the participants had an incentive, it did not provoke them to use an agenda, but they were more likely to examine the value of the item that they were selecting for study, which in turn would influence self-paced study, as described above. This explanation is post hoc, so future 
systematic investigation is needed to understand the mechanism through which reward and incentives differentially influence item selection and self-paced study.

\section{Experiment 4}

In Experiment 3, we gave some participants a monetary incentive to motivate them to construct an agenda to choose the high-reward items first for study. This monetary incentive had no effect on item selection, even when participants could only select one item for study on each trial. This outcome was surprising, because selecting the 5-point item for study was the easiest way to earn the $\$ 10$ reward, but participants on average selected the 5-point items for study only around $50 \%$ of the time. Thus, this outcome again raises the question, When participants can only choose one item for study, why do they ever select 1- and 3-point items? One answer is that participants may have incorrectly believed that item difficulty was associated with item reward in these experiments. In particular, they may have believed that the reason that some items would return a 5-point reward if recalled was because those items were more difficult to learn; if so, they may have decided not to study those more difficult items, so as to maximize their returns on recalling the lower-valued (but easier to learn) items.

To evaluate this possibility, we explicitly instructed some participants that item difficulty was not associated with reward value (debias groups). Participants in the debias groups were instructed that all items (regardless of their point values) were equally difficult to learn. To ensure that participants read the instructions, they answered the following question before beginning the task: "Is the item difficulty associated with item reward?" After responding "no" (all of the participants responded "no"), they began the task.

In addition to debiasing participants about the relationship between item difficulty and item reward, we also manipulated how many points the high-reward items were worth on each trial: Items were worth either 1,3 , and 5 points (low reward contrast) or 1, 3, and 50 points (high reward contrast). The purpose of this manipulation was to create a context in which the contrast in value was even larger, and hence would be more likely to trigger the construction of an agenda to focus study on the higher-valued items (as predicted by the first central hypothesis described above). Perhaps in this case participants would exclusively choose the 50-point items first for study.

\section{Method}

Participants A group of 125 students from KSU participated for credit in an Introductory Psychology course. A 6 (presentation order) $\times 3$ (item reward) $\times 2$ (debias: debias vs. no debias) $\times 2$ (reward contrast: low vs. high) mixed factorial design was used, with Presentation Order and Item Reward as within-subjects factors. For reward contrast, the high-valued item was either 5 points (low contrast) or 50 points (high contrast). Participants were randomly assigned to the no-debias group with low reward contrast $(n=32)$, the no-debias group with high reward contrast $(n=31)$, the debias group with low reward contrast $(n=31)$, or the debias group with high reward contrast $(n=31)$.

Materials and procedures The materials and procedure were identical to those administered in Experiment 3, with the following exceptions. First, all participants were instructed that they could only select one item on each trial for study (as in the choice-limit groups from Exps. 2 and 3). Second, all participants received the monetary incentive offered in Experiment 3. Third, we included a debias manipulation in which some participants were instructed that item difficulty is not associated with item reward, but instead that all items were equally difficult to learn. To ensure that participants read this portion of the task instructions, they were quizzed prior to starting the task. Namely, they were asked, "Is there any association between point value and item difficulty in this experiment?" and responded by clicking one of two buttons, which were labeled as "yes" and "no." All other instructions were identical to the choicelimit-with-incentive instructions from Experiment 3.

We also included a reward contrast manipulation. Participants in the low-reward-contrast groups were presented with items with a 1-, 3-, or 5-point reward. Participants in the highreward-contrast groups studied items that were worth 1,3 , or 50 points. The number of points that participants needed to earn to receive the monetary reward was also increased for this group, to account for the increased magnitude of the highreward items; participants were instructed that they would receive the $\$ 10$ reward if they earned 400 points on the final test. Note that participants could achieve this goal by recalling the same number of items as in the low contrast group (eight high-reward items). In addition, they were also instructed that their name would be entered in a raffle to win $\$ 50$ if they earned 600 points.

\section{Results and discussion}

Item selection The proportions of items selected first for study are presented in Fig. 5. A 2 (position selected: left vs. high reward) $\times 2$ (debias) $\times 2$ (reward contrast $) \times 4$ (presentation order) ANOVA revealed significant effects of position selected, $F(1,121)=81.68, M S E=30.95, \eta_{\mathrm{p}}{ }^{2}=$ .28 , and reward contrast group, $F(1,121)=7.11, M S E=$ 


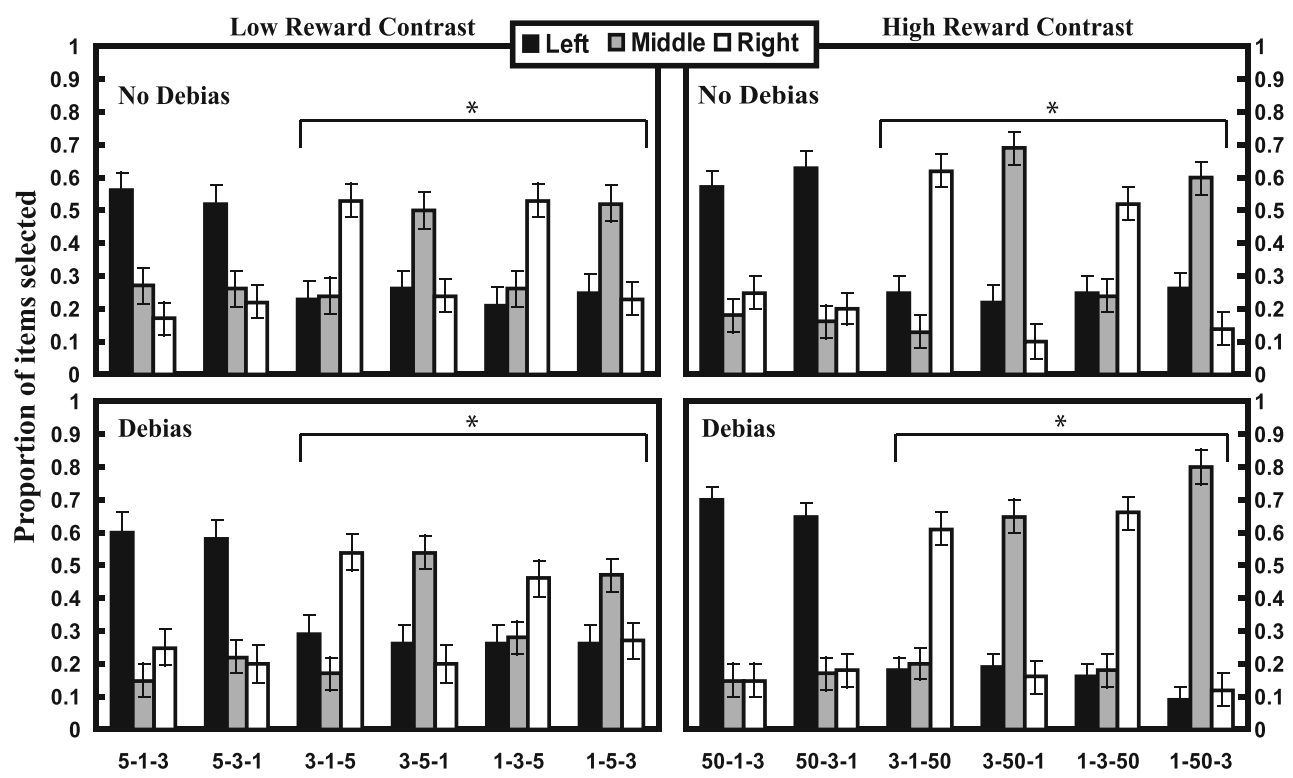

Fig. 5 Proportions of items chosen first for study in the left, middle, and right positions of the array when participants were not debiased about the relationship between item difficulty and point value and high-reward items were worth 5 points (top left panel), when they were not debiased and high-reward items were worth 50 points (top right panel), when they were debiased and high-reward items were worth 5 points (bottom left panel), and when they were debiased and

$0.38, \eta_{\mathrm{p}}{ }^{2}=.06$. These main effects were qualified by a Position Selected $\times$ Reward Contrast interaction, $F(1,121)$ $=5.54, M S E=2.10, \eta_{\mathrm{p}}{ }^{2}=.04$. This interaction occurred because participants preferred selecting items in the highreward position of the array, and this preference was magnified when the high-reward item was assigned a 50-point reward. The debias manipulation did not influence preferences for selecting items, which indicates that the results from the previous experiments were likely not due to participants believing that item difficulty was associated with item reward.

Self-paced study The mean self-paced study times are presented in Table 1. Participants in each group studied the highest-reward items (5 or 50 points) longer than the 1- or 3point items, $t \mathrm{~s}>2.71$. The debias group with low reward contrast was the only group who studied 3-point items longer than 1-point items, $t(30)=2.78, p<.01$. Consistent with these findings, a 3 (item reward) $\times 2$ (debias) $\times 2$ (reward contrast) ANOVA indicated that only the effect of item reward was significant, $F(1,120)=19.77, M S E=2.10, \eta_{\mathrm{p}}{ }^{2}=.25$.

\section{General discussion}

In the present experiments, we discovered that two conditions are likely necessary for learners to shift away from high-reward items were worth 50 points (bottom right panel) in Experiment 4. Presentation order conditions are presented on the $x$ axis: $1=1$ point, $3=$ points, $5=5$ points, $50=50$ points. Only the order conditions in brackets are relevant for contrasting the influences of agenda-based and habitual processes. Error bars represent standard errors of the means

habitual responding during study. In particular, according to one hypothesis, sufficient time must be available to construct an agenda. This hypothesis was confirmed in Experiment 1, because when learners were not pressured to make a decision quickly (vs. when they had a limited time to construct an agenda and study), they were more likely to prioritize the more highly valued items for study. According to the second hypothesis, the cost of investing time and resources to construct an agenda must be outweighed by the expected gains to performance that agenda-based regulation will yield. For instance, one condition that was particularly effective for triggering agenda construction was when the number of items that participants could select was restricted on each trial (Exps. 2-4). Under this condition, selecting items on the basis of reading order would have resulted in participants never studying the highestvalued item on trials when it was not presented in the left position of the array. Thus, constructing a value-based agenda (vs. choosing items from left to right) was an obviously more effective way to maximize performance, and when participants could only select one item on each trial, they more often, but not exclusively, preferred to select the highest-valued items.

Given the importance of selecting 5-point items first, these outcomes also raised the question, Why did learners fail to exclusively select high-reward items for study when they were only allowed to study one item on each trial? As is shown in the right panel of Fig. 3 (Exp. 2), learners could 
only select one item per trial, yet the high-valued item was selected only half the time. This suboptimal selection could not be explained by a lack of extrinsic motivation (Exps. 3 and 4) or by an inaccurate belief that highly valued items were the most difficult to learn (Exp. 4). One reason that participants might have used this less effective selection strategy was that, despite limiting the number of choices that participants could make on each trial, reading order still biased their decision making. That is, one might expect that item order alone might have undermined the use of a valuebased agenda in the present experiments, because it could bias learners to select lower-valued items when they were encountered first during reading. Though this is possible, the present results do not entirely support this conclusion, because when one excludes the position where the highestvalued item was located in the choice-limit groups, participants did not always appear to prefer selecting items in the leftmost position of the array more than items in the remaining position. For instance, consider the top right panel of Fig. 4 (Exp. 3), which is the choice-limit group with no incentive. Of the leftmost three bars, when excluding the position for the 5-point items, participants did not prefer to select those items in the leftmost position (i.e., in this case, the 1-point item in the middle position).

Another possible reason for the suboptimal regulation is that even if learners consistently constructed an agenda to focus on the highest-valued items, fatigue might have undermined executing this agenda, especially near the end of the task, when fatigue could have been a factor. We evaluated this hypothesis by examining the choice-limited groups in Experiments 2 and 3 and all groups in Experiment 4. In particular, we compared the proportions of high-reward items selected for study during the first six trials (Trials 1-6), the middle six trials (Trials 7-12), and the final six trials (13-18). If fatigue contributed to learners' selection of low-valued items when they had a choice limit, high-reward items should be selected less often in the later block of trials. In contrast to this prediction, no significant differences occurred in the proportions of high-reward items selected for study across blocks of trials in any experiment (in Exp. 2, early trials $M=$ $.47, S E=.06$; middle $M=.47, S E=.07$; late $M=.48, S E=.06$; in Exp. 3, early $M=.41, S E=.04$; middle $M=.48, S E=.04$; late $M=.43, S E=.04$; in Exp. 4, early $M=.56, S E=.04$; middle $M=.61, S E=.03$; late $M=.60, S E=.03), F \mathrm{~S}<1.4$.

A final reason considered here is that individual differences in a fundamental cognitive capacity (e.g., working memory capacity or attentional deficits) could have undermined effective agenda construction and execution for learners with lower abilities (Ariel et al., 2009; Dunlosky \& Thiede, 2004; for other individual differences that may have contributed, see Dunlosky \& Ariel, 2011b). As was reported by Dunlosky and Thiede (2004), individuals with lower working memory spans (vs. higher spans) show a dual cost to the effective allocation of study time: (a) They often do not construct an effective agenda, even under relatively obvious conditions, and (b) when they are given an agenda to use, they do not consistently execute it during study (for other supporting evidence, see Castel, Lee, Humphreys, \& Moore, 2011).

The relationship between agenda-based and habitual processes

The present experiments indicate that learners' study decisions can be influenced by either agenda-based or habitual processes. How these processes operate in relation to each other is unclear. One possibility is that each process exerts an exclusive influence over each learner's study decisions, with each learner either always using an agenda or never using one. In this case, if task conditions trigger the use of agenda-based processes for a given individual, these processes would completely override the influence of habitual processing on study decisions across all trials. Another possibility is that task conditions that promote agenda construction simply attenuate the influence of habitual processing by reducing the likelihood that habitual processes influence study decisions on any given trial.

To illustrate, consider differences between the no-choicelimit groups and the choice-limit groups. If agenda-based and habitual processes operate in an exclusive manner, then most learners should select items in the left position of the array on every trial when they study without a choice limit.

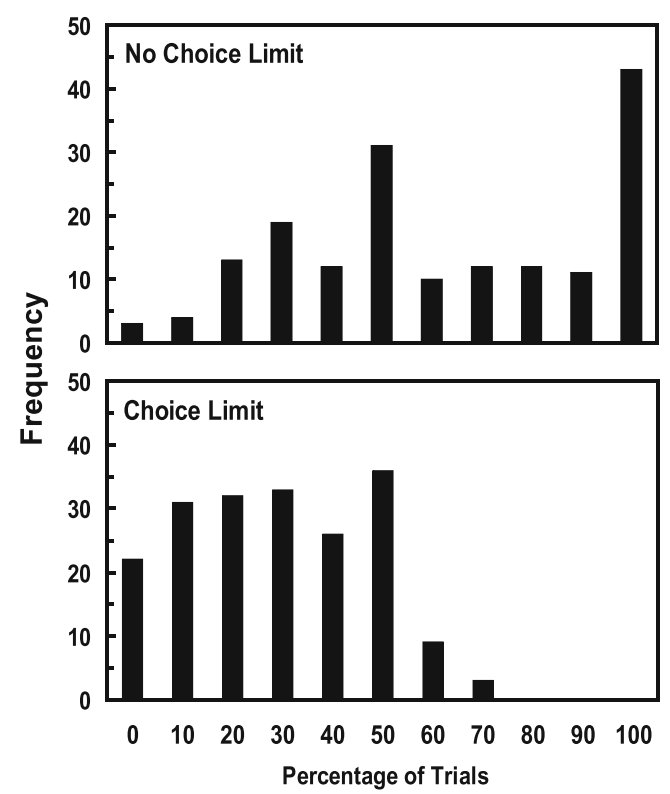

Fig. 6 Numbers of individuals (or frequency) in the no-choice-limit groups (top panel) and the choice-limit groups (bottom panel) from Experiments 1-4 who selected items in the left position of the array first for study (excluding the 5-1-3 and 5-3-1 conditions) on $0 \%$, $10 \%, 20 \%, 30 \%, 40 \%, 50 \%, 60 \%, 70 \%, 80 \%, 90 \%$, and $100 \%$ of trials 
Most importantly, when participants can select only one item per trial, more individuals should never select items in the left position of the array when it is not occupied by the high-reward item; that is, the individuals who reacted to the choice limit by developing an agenda should exclusively use that agenda (selecting 5-point items) on every trial. In contrast, if agenda-based and habitual processes do not operate in an exclusive manner, then task conditions that promote agenda construction (such as limiting the number of choices per trial) would not completely eliminate the influence of habitual processes on study decisions.

We evaluated these predictions about the relationship between agenda-based and habitual processes by computing the percentages of trials on which each participant selected items at the left position of the array first for study when the left position was not occupied by the high-reward item (i.e., the 5-1-3 and 5-3-1 conditions were excluded). We then computed frequencies with these data to examine the distribution of the percentages of trials on which study decisions were influenced by habitual processes. We computed these frequencies separately for individuals in the no-choice-limit and choice-limit groups, because this factor was the most effective at promoting agendabased regulation, and collapsing across experiments yielded a large sample size (no choice limit, $N=170$; choice limit, $N=$ 192). We collapsed across the other manipulations because they had a minimal influence on item selection.

The frequency data presented in Fig. 6 reveal that when participants were not given a choice limit (top panel of Fig. 6), a large proportion of individuals selected items in a manner consistent with habitual responding. In fact, $25 \%$ of individuals (43 total) selected items in the left position of the array first for study on $100 \%$ of the trials, and $46 \%$ of individuals (78 total) selected items in the left position on $70 \%$ or more of the trials. In contrast, when learners were instructed that they could only select one item for study on each trial (bottom panel of Fig. 6), $11 \%$ (22 total) never selected items in the left position of the array, which could indicate that for these individuals agenda-based and habitual processes exerted exclusive influences. However, selection behavior for the majority of participants in the choice-limit group was inconsistent with this possibility, since the majority ( $89 \%$, or 170 total) selected items in the left position on at least $10 \%$ of the trials. These data suggest that factors that trigger agenda-based processes (e.g., choice limits) attenuate the influence of habitual processes on individual learners' study decisions without completely diminishing that influence. Thus, agenda-based and habitual processes do not operate in an exclusive manner.

\section{Summary}

In the present experiments, we systematically investigated the degrees to which a variety of factors could trigger agenda use, and hence undermine the influence of habitual processes on study decisions. As expected, conditions that limited the effectiveness of a left-to-right selection bias increased the likelihood that learners would prioritize highvalue items for study. Perhaps surprisingly, even under these conditions, learners did not consistently select items for study that would yield the highest potential reward.

\section{References}

Ariel, R., Al-Harthy, I. S., Was, C. A., \& Dunlosky, J. (2011). Habitual reading biases in the allocation of study time. Psychonomic Bulletin and Review, 18, 1015-1021. doi:10.3758/s13423-011-0128-3

Ariel, R., Dunlosky, J., \& Bailey, H. (2009). Agenda-based regulation of study-time allocation: When agendas override item-based monitoring. Journal of Experimental Psychology. General, 138, 432 447. doi: $10.1037 / \mathrm{a} 0015928$

Baumeister, R. F., \& Heatherton, T. F. (1996). Self-regulation failure: An overview. Psychological Inquiry, 7, 1-15.

Castel, A. D. (2007). The adaptive and strategic use of memory by older adults: Evaluative processing and value-directed remembering. In A. S. Benjamin \& B. H. Ross (Eds.), The psychology of learning and motivation: Skill and strategy in memory use (Vol. 48, pp. 225-270). San Diego: Academic Press.

Castel, A. D., Balota, D. A., \& McCabe, D. P. (2009). Memory efficiency and the strategic control of attention at encoding: Impairments of value-directed remembering in Alzheimer's disease. Neuropsychology, 23, 297-306.

Castel, A. D., Benjamin, A. S., Craik, F. I. M., \& Watkins, M. J. (2002). The effects of aging on selectivity and control in shortterm recall. Memory and Cognition, 30, 1078-1085. doi:10.3758/ BF03194325

Castel, A. D., Lee, S. S., Humphreys, K. L., \& Moore, A. N. (2011). Memory capacity, selective control, and value-directed remembering in children with and without attention-deficit/hyperactivity disorder. Neuropsychology, 25, 15-24.

Chaiken, S. (1980). Heuristic versus systematic information processing and the use of source versus message cues in persuasion. Journal of Personality and Social Psychology, 39, 752-766.

De Neys, W. (2006). Dual processing in reasoning: Two systems but one reasoner. Psychological Science, 17, 428-433.

Devine, P. G. (1989). Stereotypes and prejudice: Their automatic and controlled components. Journal of Personality and Social Psychology, 56, 5-18.

Dunlosky, J., \& Ariel, R. (2011a). The influence of agenda-based and habitual processes on item selection during study. Journal of Experimental Psychology: Learning, Memory, and Cognition, 37, 899-912. doi: $10.1037 / \mathrm{a} 0023064$

Dunlosky, J., \& Ariel, R. (2011b). Self-regulated learning and the allocation of study time. In B. H. Ross (Ed.), The psychology of learning and motivation (Vol. 54, pp. 103-140). San Diego: Academic Press.

Dunlosky, J., \& Thiede, K. W. (1998). What makes people study more? An evaluation of factors that affect people's self-paced study and yield "labor-and-gain" effects. Acta Psychologica, 98, 37-56.

Dunlosky, J., \& Thiede, K. W. (2004). Causes and constraints of the shift-to-easier-materials effect in the control study. Memory and Cognition, 32, 779-788.

Evans, J. S. B. T. (2003). In two minds: Dual-process accounts of reasoning. Trends in Cognitive Sciences, 7, 454-459. doi:10.1016/ j.tics.2003.08.012 
Evans, J. S. B. T., \& Curtis-Holmes, J. (2005). Rapid responding increases belief bias: Evidence for the dual-process theory of reasoning. Thinking and Reasoning, 11, 382-389.

Evans, J. S. B. T., \& Frankish, K. (Eds.). (2009). In two minds: Dual processes and beyond. Oxford: Oxford University Press.

Hayes-Roth, B., \& Hayes-Roth, F. (1979). A cognitive model of planning. Cognitive Science, 3, 275-310.

Isbell, L. M., \& Wyer, R. S., Jr. (1999). Correcting for mood-induced bias in evaluations of political candidates: The roles of intrinsic and extrinsic motivation. Personality and Social Psychology Bulletin, 25, 237-249.

Kahneman, D., \& Peavler, W. S. (1969). Incentive effects and pupillary changes in association learning. Journal of Experimental Psychology, 79, 312-318. doi:10.1037/h0026912

Kool, W., McGuire, J. T., Rosen, Z. B., \& Botvinick, M. M. (2010). Decision making and avoidance of cognitive demand. Journal of Experimental Psychology. General, 139, 665-682.

Metcalfe, J. (2002). Is study time allocated selectively to a region of proximal learning? Journal of Experimental Psychology. General, 131, 349-363. doi:10.1037/0096-3445.131.3.349

Metcalfe, J. (2009). Metacognitive judgments and control of study. Current Directions in Psychological Science, 18, 159-163. doi:10.1111/j.1467-8721.2009.01628.x

Metcalfe, J., \& Kornell, N. (2005). A region of proximal learning model of study time allocation. Journal of Memory and Language, 52, 463-477.

Metcalfe, J., \& Mischel, W. (1999). A hot/cool-system analysis of delay of gratification: Dynamics of willpower. Psychological Review, 106, 3-19.

Nelson, T. O., \& Leonesio, R. J. (1988). Allocation of self-paced study time and the "labor-in-vain effect. Journal of Experimental Psychology: Learning, Memory, and Cognition, 14, 676-686. doi:10.1037/0278-7393.14.4.676

Petty, R. E., \& Cacioppo, J. T. (1986). The elaboration-likelihood model of persuasion. Advances in Experimental Social Psychology, 19, 123-205.

Pintrich, P. R. (2000). The role of goal orientation in self-regulated learning. In M. Boekaerts, P. R. Pintrich, \& M. Zeidner (Eds.), Handbook of self-regulation (pp. 451-502). New York: Academic Press.
Pintrich, P. R., \& De Groot, E. (1990). Motivational and self-regulated learning components of classroom academic performance. Journal of Educational Psychology, 82, 33-40.

Price, J., \& Murray, R. G. (2012). The region of proximal learning heuristic and adult age differences in self-regulated learning. Psychology and Aging. doi:10.1037/a0029860

Roberts, M. J., \& Newton, E. J. (2002). Inspection times, the change task, and the rapid-response selection task. The Quarterly Journal of Experimental Psychology, 54A, 1031-1048.

Schroyens, W., Schaeken, W., \& Handley, S. (2003). In search of counter-examples: Deductive rationality in human reasoning. The Quarterly Journal of Experimental Psychology, 56, 11291145.

Soderstrom, N. C., \& McCabe, D. P. (2011). The interplay between value and relatedness as bases for metacognitive monitoring and control: Evidence for agenda-based monitoring. Journal of Experimental Psychology: Learning, Memory, and Cognition, 37, 1236-1242.

Son, L. K., \& Metcalfe, J. (2000). Metacognitive and control strategies in study-time allocation. Journal of Experimental Psychology: Learning, Memory, and Cognition, 26, 204-221.

Teasdale, J. D. (1999). Multi-level theories of cognition-emotion relations. In T. Dalgleish \& M. J. Power (Eds.), Handbook of cognition and emotion (pp. 665-681). Chichester: Wiley.

Thiede, K. W., \& Dunlosky, J. (1999). Toward a general model of selfregulated study: An analysis of selection of items for study and self-paced study time. Journal of Experimental Psychology: Learning, Memory, and Cognition, 25, 1024-1037.

van Reekum, C. M., \& Scherer, K. R. (1997). Levels of processing for emotion-antecedent appraisal. In G. Matthews (Ed.), Cognitive science perspectives on personality and emotion (pp. 259-300). Amsterdam: Elsevier.

Waldron, S. M., Patrick, J., \& Duggan, G. B. (2011). The influence of goal-state access cost on planning during problem solving. The Quarterly Journal of Experimental Psychology, 64, 485-503. doi:10.1080/17470218.2010.507276

Winne, P. H., \& Hadwin, A. F. (1998). Studying as self-regulated learning. In D. J. Hacker, J. Dunlosky, \& A. C. Graesser (Eds.), Metacognition in educational theory and practice (pp. 277-304). Hillsdale: Erlbaum. 